\title{
The anterior-posterior laxity after total knee arthroplasty inserted with a ligament tensor
}

\author{
C. T. H. van Hal · G. G. van Hellemondt • \\ A. B. Wymenga $\cdot$ W. C. H. Jacobs
}

Received: 19 January 2007 / Accepted: 16 March 2007/Published online: 17 April 2007

(C) Springer-Verlag 2007

\begin{abstract}
Goal of this study is to determine the anteriorposterior laxity in $30^{\circ}$ of knee flexion for a posterior cruciate retaining total knee arthroplasty with a relative dished insert and implanted with a ligament tensor. Furthermore, the correlation between these AP laxities and the postoperative range of motion (ROM) and postoperative Knee Society Score (KSS) is analysed. Fifty-one balanSys ${ }^{\mathrm{TM}}$ total knee arthroplasties were performed in 49 patients between 1998 and 2000. These arthroplasties are analysed with respect to AP laxity (Rolimeter), ROM and KSS with a mean follow-up of 4.6 years. The mean anterior laxity is $2.8 \mathrm{~mm}$ with no posterior laxities at all. The average postoperative ROM is $110^{\circ}$ with an average KSS of 142 . No correlations between AP-laxity and postoperative ROM or between AP-laxity and postoperative KSS are found. A posterior cruciate retaining TKA with a relative dished insert and implanted with a tensor is very stable in the anterior-posterior direction in $30^{\circ}$ of knee flexion. This limited laxity does not seem to disadvantage the mean postoperative ROM and KSS, when compared to other TKA studies.
\end{abstract}

Keywords Knee $\cdot$ TKA $\cdot$ Laxity $\cdot$ Sagittal $\cdot$ Rolimeter

C. T. H. van Hal - G. G. van Hellemondt .

A. B. Wymenga - W. C. H. Jacobs

Department of Orthopedics, Sint Maartenskliniek,

Nijmegen, The Netherlands

W. C. H. Jacobs $(\bowtie)$

Orthopedic Research Unit, Department of Research, Development and Education, Sint Maartenskliniek, Postbus 9011, 6500 GM Nijmegen, The Netherlands

e-mail: w.jacobs@maartenskliniek.nl

\section{Introduction}

In literature there is controversy about the 'laxity' of a TKA and its relation with the postoperative achieved patient satisfaction, postoperative ROM and knee functioning. For example, where Yamakado et al. [16] show no correlation between the anterior-posterior laxity with the post-operative ROM, Itokazu et al. [8] did found a correlation with the postoperative ROM. Hypothetically, a loose total knee arthroplasty is thought to result in a better ROM compared to a tight knee [15]. However, a loose arthroplasty might also create more problems with respect to instability causing pain and dysfunctioning. Moreover, instability due to ligament laxity is an important factor in the cause of PE wear. PE wear is related to the way the dished insert compensates for presence of instability. Instability due to absence of the PCL could be compensated by a dished insert. With respect to the most optimal laxity, Ischii et al. [7] and Pellengahr et al. [12] show best postoperative results in total knee arthroplasties with anterior laxities less than $6 \mathrm{~mm}$. However, Warren et al. [15] found more extension deficits and less ROM when the anterior laxity is below $5 \mathrm{~mm}$.

The ligament tension and geometry of the polyethylene insert may influence the AP laxity with less tension of the ligaments creating more AP laxity [14]. A more dished insert means more congruency with the femoral component, leading to less AP laxity in the knee. This also makes the prosthesis more constrained. Laz et al. [9] showed more AP laxity for an unconstrained prosthesis compared to a semi-constrained prosthesis. Since 1998 we started to use a ligament guided knee system (balanSys ${ }^{\mathrm{TM}}$ ) with a calibrated tensor and a relatively dished insert. A relatively dished insert makes this unconstrained implant design somewhat constrained. Goal of this study was to determine 
the anterior-posterior laxity in $30^{\circ}$ of knee flexion in this type of implant with $150 \mathrm{~N}$ tension applied in extension and flexion during implantation of the knee. The $30^{\circ}$ position is chosen because this is the most lax position in the knee. This will be done with a mean follow-up of 4.6 years. The correlation between these anterior-posterior laxities and the postoperative ROM and postoperative Knee Society Score (KSS) is also analysed.

\section{Methods}

TKA: BalanSys ${ }^{\mathrm{TM}}$

The balanSys ${ }^{\mathrm{TM}}$ cruciate retaining total knee system (Mathys Ltd, Bettlach, Switzerland) is implanted with a ligament-referenced technique. After the tibiacut the knee is aligned in extension with ligament releases if needed. After this, the knee is balanced in flexion with the force controlled tensor $(150 \mathrm{~N})$ and the bonecuts are based on the ligament tension. Next step is the extension cut of the femur also guided by ligament tension $(150 \mathrm{~N})$. This unconstrained knee prosthesis is called the 'balanSys ${ }^{\mathrm{TM}}$ ' total knee arthroplasty. In this way the created extension and flexion gaps are equal. This should optimize the tensioning of the collateral ligaments around the knee and improve the knee stability in extension as well as in flexion (Fig. 1). The balanSys ${ }^{\mathrm{TM}}$ total knee prosthesis includes a relatively dished insert.

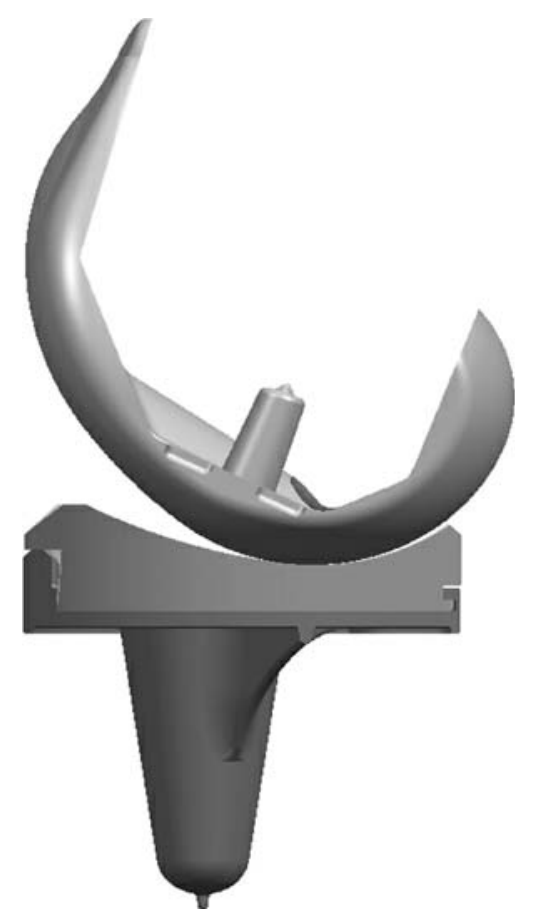

Fig. 1 Lateral aspect of the balanSys ${ }^{\mathrm{TM}}$ total knee arthroplasty components in $20^{\circ}$ of flexion
Patients

Fourty-one patients from the Sint Maartenskliniek (Nijmegen, The Netherlands) with a primary balanSys ${ }^{\mathrm{TM}}$ TKA were seen for their 5 years follow-up visit at the outpatients'clinic. These 49 patients remained from the first group of 100 consecutive patients receiving a balanSys TKA. The total knee arthroplasties were performed between September 1998 and March 2000.

Before the follow-up visit, the patients gave their informed consent. The medical ethical committee approved the study design. At the outpatients'clinic, an independent observer saw the patients.

TKA evaluation including the anterior-posterior laxity

The clinical outcome parameters include the post-operative ROM, the KSS and the AP stability of the knee in $30^{\circ}$ of flexion measured by a Rolimeter [11]. Furthermore, AP and lateral knee X-rays are performed to detect any radiolucencies. Pre-operative ROM and KSS were well documented in the patients reports. The complications were searched in the patients' surgical and outpatient clinic reports.

\section{Statistics}

For the anterior-posterior laxities mean values and standard deviations will be presented. Spearman correlations are used to analyse the correlation between these anteriorposterior laxities and the postoperative ROM and postoperative Knee Society Score (KSS).

\section{Results}

\section{Patients}

The 51 balanSys ${ }^{\mathrm{TM}}$ total knee arthroplasties were performed in 49 patients, 2 patients (women) received bilateral total knee prostheses. The indication for which they received their TKA was primary osteoarthritis in all cases. The mean age of this group was $72.5 \pm 4.4$ years (range 59-87) at the time of surgery with a mean BMI of $29.1 \pm 4.8$. The group exists of 12 men and 37 women, where 20 total knee arthroplasties were performed at the left and 31 arthroplasties at the right side. With the balancing method, 18 knees did not have any ligament release at all, 15 had a release on the medial side, 12 on the lateral, 4 at both sides and for 1 prosthesis the type of ligament release is unknown. The mean follow-up period is 4.6 years (range $4.1-5.4$ ). 


\section{TKA evaluation}

The mean post-operative knee flexion was $109.6^{\circ} \pm 16.4^{\circ}$. Twelve out of 51 TKA knees had a postoperative extension deficit of less than $5^{\circ}$, the other TKA knees did not have an extension deficit at all. There were no knees that hyperextended. The total ROM showed an average of $109.5^{\circ} \pm 17.5^{\circ}$. For the KSS, the mean score 4.6 years post-operative was $142 \pm 30$. This score is divided in a functional knee score of $51 \pm 25$ and a clinical kneescore of $91 \pm 8$. The radiological evaluation on AP and lateral knee X-rays showed no progressive radiolucencies of more than $2 \mathrm{~mm}$ in the $\mathrm{X}$-rays.

\section{Anterior-posterior laxity}

The anterior-posterior laxity determined with the Rolimeter showed a mean anterior laxity of $2.8 \pm 1.1 \mathrm{~mm}$ (range $0-5 \mathrm{~mm}$ ) and no posterior laxity at all. With respect to the anterior laxity, 4 knees had just 0 or $1 \mathrm{~mm}$ anterior laxity, where $47 \mathrm{knees}$ had an anterior laxity of 2-5 mm. Spearman correlations show no relation at all between the AP-laxity and the postoperative ROM $(r=0.12)$ or between the AP-laxity and the postoperative $\mathrm{KSS}(r=0.30)$.

\section{Discussion}

The average anterior laxity of $2.8 \mathrm{~mm}$ found for the balanSys $^{\mathrm{TM}}$ total knee arthroplasty in $30^{\circ}$ of flexion, seems small when comparing it with other studies. In $30^{\circ}$ of knee flexion, average anterior laxities between 2.9 and $5.3 \mathrm{~mm}$ are found for other total knee arthroplasties [2, 7, 12]. For the posterior laxity of the balanSys ${ }^{\mathrm{TM}}$ TKA, no knee laxity at all was found in this study. Average posterior laxities of 1.1 to $1.9 \mathrm{~mm}$ were presented in comparable studies [7, 12]. They included respectively PCL retaining prostheses [2], both PCL retaining and substituting prostheses [7] and unconstrained prostheses [12].

These studies $[2,7,12]$ were performed with the Telos stress-X method, the KT-1000 and the KT-2000 arthrometer and were 4-6 years postoperative. In this study, the Rolimeter instrument is used to determine the anteriorposterior laxity of the TKA knee. Several studies have shown the rolimeter to be a valid and reliable testing device for the anterior-posterior translation of the knee joint [5, $11,13]$ in the $90^{\circ}$ as well as the $30^{\circ}$ of knee flexion [6] with no significant difference between Rolimeter measurements compared to other testing devices [1].

The AP laxity of a total knee arthroplasty is determined by the absence or presence of the ACL, PCL, the tensioning of the collateral ligaments and the type of insert. The PCL function however, is more present in the $60^{\circ}-120^{\circ}$ of knee flexion and is of less importance in $30^{\circ}$. The $30^{\circ}$ AP stability thus mainly depends on the collateral tensioning and the type of insert. We hypothesis that a better collateral ligament tensioning achieved by the force controlled tensor could be responsible for a better femoral component fit in its insert which will reduce translations in the anteriorposterior direction.

Cloutier et al. [3] found that $89 \%$ of total knee arthroplasties have an anterior laxity less than $5 \mathrm{~mm}$. The other $11 \%$ had a laxity between 5 and $10 \mathrm{~mm}$. Normal knees without a total knee arthroplasty and without an anterior cruciate ligament deficiency show average anterior laxities of 5.5 and $5.7 \mathrm{~mm}$ in $30^{\circ}$ of knee flexion [4, 10]. It is however, not clear whether a total knee arthroplasty should hypothetically have the same anterior and posterior laxities as a control knee to achieve best postoperative success. We think however, that more stable knees will result in less PE wear due to the reduction of shear forces in the polyethylene. With a stable knee we mean stability created by good ligament tensioning combined with a certain degree of implant congruency (relatively deep dish) not by mechanical constrained due to implant design alone.

The total ROM for the balanSys ${ }^{\mathrm{TM}}$ TKA in this study is $109.5^{\circ} \pm 17.5^{\circ}$, which is comparable with other TKA studies $[3,12,16]$. In these studies a total ROM of $112^{\circ}$, $107^{\circ}$ and $103.5^{\circ}$ were reported. No correlation was found in their studies between the anterior laxity in $30^{\circ}$ of flexion and the postoperative ROM and we could confirm this. This was also what we expected to find. Itokazu et al. however, found better flexion in patients with a larger AP laxity.

With respect to the KSS, the average of 142.5 of the balanSys ${ }^{\mathrm{TM}}$ TKA is comparable with the average KSS of 152.2 found by Pellengahr [12]. For the KSS, there is also no correlation with the anterior knee laxity.

As mentioned before in the methods 49 out of the first 100 patients receiving a balanSys TKA were included in this study. From the selected group of 100 patients unfortunately just 65 patients with 67 arthroplasties were able and willing to come to the outpatients'clinic. Main reason will be the mean age at time of follow-up above 75 years. For another 16 knees the AP laxity could not be measured accurately because of (extreme) adipositas, a lot of pain or comorbidity like a spastic component due to a cerebrovascular accident. This is why only 51 knees are left for analyses in this study.

With a mean anterior displacement of $2.8 \mathrm{~mm}$ and no posterior displacement at all, the cruciate retaining balanSys $^{\mathrm{TM}}$ total knee arthroplasty with a relative dished insert has shown to be very stable one in anterior-posterior direction. Despite this AP stability, a good postoperative ROM and a good KSS are found similar to other cruciate retaining knee systems. 


\section{References}

1. Balasch H, Schiller M, Friebel H, Hoffmann F (1999) Evaluation of anterior knee joint instability with the Rolimeter. A test in comparison with manual assessment, measuring with the KT-1000 arthrometer. Knee Surg Sports Traumatol Arthrosc 7(4):204-208

2. Besson A, Brazier J, Chantelot C, Migaud H, Gougeon F, Duquennoy A (1999) Laxity and functional results of MillerGalante total knee prosthesis with posterior cruciate ligament sparing after a 6-year follow-up. Rev Chir Orthop Reparatrice Appar Mot 85(8):797-802

3. Cloutier JM, Sabouret P, Deghrar A (1999) Total knee arthroplasty with retention of both cruciate ligaments. A nine to elevenyear follow-up study. J Bone Joint Surg Am 81(5):697-702

4. Daniel DM, Malcom LL, Losse G, Stone ML, Sachs R, Burks R (1985) Instrumented measurement of anterior laxity of the knee. J Bone Joint Surg Am 67(5):720-726

5. Ganko A, Engebretsen L, Ozer H (2000) The rolimeter: a new arthrometer compared with the KT-1000. Knee Surg Sports Traumatol Arthrose 8(1):36-39

6. Hatcher J, Hatcher A, Arbuthnot J, McNicholas M (2005) An investigation to examine the inter-tester and intra-tester reliability of the Rolimeter knee tester, and its sensitivity in identifying knee joint laxity. J Orthop Res 23(6):1399-1403

7. Ishii Y, Matsuda Y, Ishii R, Sakata S, Omori G (2005) Sagittal laxity in vivo after total knee arthroplasty. Arch Orthop Trauma Surg 125(4):249-253

8. Itokazu M, Masuda K, Wada E, Ohno T, Yoshida M, Takatu T (1987) Influence of anteroposterior and mediolateral instability on range of motion after total knee arthroplasty: an ultrasonographic study. Clin Orthop 219:147-157

9. Laz PJ, Pal S, Fields A, Petrella AJ, Rullkoetter PJ (2006) Effects of knee simulator loading and alignment variability on predicted implant mechanics: A probabilistic study. J Orthop Res Sep 26 (Epub ahead of print)

10. Markolf KL, Kochan A, Amstutz HC (1984) Measurement of knee stiffness and laxity in patients with documented absence of the anterior cruciate ligament. J Bone Joint Surg Am 66(2):242252

11. Muellner T, Bugge W, Johansen S, Holtan C, Engebretsen L (2001) Inter- and intratester comparison of the Rolimeter knee tester: effect of tester's experience and the examination technique. Knee Surg Sports Traumatol Arthrosc 9(5):302-306

12. Pellengahr C, Jansson V, Durr HR, Refior HJ (1999) Significance of sagittal stability in knee prosthesis implantation-an analysis of 76 cases with unconstrained joint surface replacement. Z Orthop Ihre Grenzgeb 137(4):330-333

13. Schuster AJ, McNicholas MJ, Wachtsl SW, Mc Gurthy DW, Jakob RP (2004) A new mechanical testing device for measuring anteroposterior knee laxity. Am J Sports Med 32(7):1731-1735

14. Wada M, Tatsuo H, Kawahara H, Sato M, Baba H (2001) In vivo kinematic analysis of total knee arthroplasty with four different polyethylene designs. Artif Organs 25(1):22-28

15. Warren PJ, Olanlokun TK, Cobb AG, Walker PS, Iverson BF (1994) Laxity and function in knee replacements. A comparative study of three prosthetic designs. Clin Orthop 305:200-208

16. Yamakado K, Kitaoka K, Yamada H, Hashiba K, Nakamura R, Tomita K (2003) Influence of stability on range of motion after cruciate-retaining TKA. Arch Orthop Trauma Surg 123(1):1-4 